\title{
RESEARCH HIGHLIGHT UIM-UDS: a new interface between ATG8 and its interactors
}

\author{
Yuchen Lei ${ }^{1}$ and Daniel J. Klionsky (D) ${ }^{1}$ \\ Cell Research (2019) 29:507-508; https://doi.org/10.1038/s41422-019-0179-y
}

\begin{abstract}
The autophagy-related protein ATG8 (mammalian LC3) plays a key role in cargo recognition during selective macroautophagy/autophagy. The ubiquitin-interacting motif (UIM)-UIMdocking site (UDS) was recently identified as a new interface between ATG8 and its interactors, which expands the list of autophagy receptors and adaptors, and also suggests an additional avenue for potential therapeutic designs for the treatment of human diseases.
\end{abstract}

Autophagy is a conserved process in eukaryotic cells, during which subcellular components or macromolecules are sequestered and then transported to the vacuole or lysosome for degradation and recycling. ${ }^{1}$ Autophagy can be classified as nonselective, which is usually used to replenish energy and/or metabolites under nutrient-starvation conditions, or selective. Selective autophagy refers to the specific degradation of organelles, microbes or proteins. ${ }^{2}$ Various autophagy receptors have been identified to mediate the sequestration of specific cargos during selective autophagy. The significance of this process is evident in certain diseases caused by the inefficient or blocked clearance of specific subcellular components. ${ }^{3}$

Autophagy begins with the formation of a double-membrane compartment called the phagophore that sequesters appropriate cargos, subsequently maturing into an autophagosome; the latter ultimately fuses with the vacuole/lysosome to deliver the contents to this recycling organelle. One of the core events of this process as first described in yeast is Atg8 conjugation to the lipid phosphatidylethanolamine (PE) with the help of other Atg proteins such as Atg7, Atg3, and the Atg12-Atg5 complex, ${ }^{4}$ after which Atg8-PE will attach to the phagophore membrane. Atg8 is not only important for phagophore expansion, ${ }^{5}$ but also provides a docking site for autophagy receptors. Among other functions, the existence of these receptors connects ubiquitinated proteins and autophagy, enabling their selective degradation.

Many yeast/plant proteins that bind Atg8/ATG8 have an Atg8interacting motif (AIM; similar to the LC3-interacting region (LIR) in mammalian cells); a conserved sequence in the AIM can be recognized by hydrophobic pockets on Atg8, usually called the LIR/AIM-docking site (LDS). ${ }^{6}$ Although many studies have focused on the AIM-LDS interface, the possibility remains that there are other binding sites on Atg8 as well as on autophagy receptors. Recently, Marshall et al. described a new interface between plant ATG8 and its interactors and identified a wide range of receptors and adaptors that bind to ATG8 through this noncanonical mechanism. ${ }^{7}$

In their previous work, ${ }^{8}$ the authors found that in Arabidopsis, RPN10 functions as an autophagy receptor for the ubiquitinated $26 \mathrm{~S}$ proteasome; however, its binding to ATG8 depends on ubiquitin-interacting motifs (UIMs), not the canonical AIM. The discovery of a UIM on RPN10 provided a hint that there may be domains on ATG8, other than the LDS, involved in the binding with certain cargo. By mapping conserved sequences in plant ATG8 to the three-dimensional structure of yeast Atg8, they found an additional eight possible binding sites for the UIM domain besides the LDS. Alanine substitution of these sites, coupled with binding assays, revealed that the UIM-docking site (UDS) is involved in the binding between ATG8 and RPN10. Impaired binding between ATG8 $\triangle$ UDS and RPN10 further confirmed that the UDS participates in the RPN10-ATG8 interaction. Additional experiments showed that the UDS is characterized by a conserved phenylalanine surrounded by hydrophobic residues, and that the UDS is located opposite to the LDS on ATG8, which explains why AIM- and UIM-containing proteins can bind to ATG8 simultaneously.

An obvious question is whether the UIM is important for RPN10 only or if it is used more widely. A binding assay examining thirteen Arabidopsis proteins predicted to have UIM sequences showed that three plant ubiquitin regulatory $X$ domain (PUX) proteins have a strong interaction with ATG8. Through further screening of all proteins in the PUX family, PUX7_ARATH/AT1G14570, PUX8_ARATH/AT4G11740.1, PUX9_ARATH/AT4G00752.1 and PUX13_ARATH/ AT4G23040.1 were found to interact with ATG8, and further assays using an altered UIM and ATG8 $\triangle$ UDS confirmed that the UIM-UDS interaction is necessary for their binding. In addition, proteins that are not annotated to have a UIM still have the possibility to bind with ATG8 through this novel interface. To find out whether there are other ATG8 interactors, an unbiased screen of an Arabidopsis cDNA library was conducted, and 112 potential ATG8 interactors were found, among which nineteen are confirmed UDS interactors.

This UIM-UDS interface exists not only in Arabidopsis, but is also conserved in yeast and human. Ent 1 and Ent2, as well as Ubx5, in yeast bind to Atg8 through this interface. Further unbiased analysis showed 17 UDS interactors in yeast. Similarly, by testing UIM-expressing proteins, they also found UDS interactors in human. All these results showed that the UIM-UDS interface is conserved, and that it is a widely used mechanism for binding between Atg8-family proteins and their interactors.

The significance of this newly found binding domain on autophagy receptors and adaptors was explored. Arabidopsis PUX proteins can interact with ATG8 through the UIM-UDS interface; therefore the authors took PUX7 and its binding substrate CDC48/AT3G09840 as an example to reveal the importance of the UIM-UDS interaction. When CDC48 is inactivated by its inhibitor or has disease-related mutations, it can become an autophagy substrate. However, differently from nitrogen starvation-induced autophagy, in which PUX proteins are not required, degradation of inactive or mutant CDC48 depends on PUX proteins, which interact with ATG8 through the

\footnotetext{
${ }^{1}$ Life Sciences Institute and Department of Molecular, Cellular and Developmental Biology, University of Michigan, Ann Arbor, MI 48109, USA

Correspondence: Daniel J. Klionsky (klionsky@umich.edu)
}

Published online: 20 May 2019 
UIM-UDS interface, suggesting that they may act as a receptor for dysfunctional CDC48. Similarly, in yeast, Ubx5, an ortholog of PUX proteins, is necessary for the degradation of dysfunctional Cdc48. Non-functional Cdc48 can bind to the UBX domain on Ubx5 and then be directed to the autophagy pathway through a UIM-UDS-mediated interaction between Atg8 and Ubx5. In both PUX-deleted Arabidopsis and UBX5-deleted yeast, growth defects appear when CDC48/Cdc48 loses its function, and these yeasts are also more sensitive to endoplasmic reticulum stress. CDC48 plays an essential role in protein quality control, and mutation of human $\mathrm{VCP} / \mathrm{p} 97 / \mathrm{CDC} 48$ can cause many diseases; ${ }^{9}$ thus, determining that the UIM-UDS interaction is essential in removing dysfunctional CDC48 may have medical significance in VCP/CDC48-related disease treatment.

In summary, based on their previous discovery of a new ATG8binding domain on RPN10, the Vierstra lab found the UIM-UDS interface as a new and widely existing interaction between ATG8 and its binding partners. This finding thus enlarges the range of autophagy receptors and adaptors, and identifies a higher complexity in autophagy cargo selection. Besides, the discovery that the UIM-UDS interaction is necessary to target non-functional CDC48 to autophagy, which is different from what happens under starvation conditions, indicates that two autophagy pathways can participate in CDC48 degradation. However, how cells distinguish between these two pathways or whether there are any specific signals that direct proteins to either pathway still remains to be answered. The fact that dysfunctional VCP/CDC48 is involved in many kinds of diseases demonstrates the importance of the UIMUDS in cell homeostasis and provides new possibilities in disease therapy.

\section{REFERENCES}

1. Xie, Z. \& Klionsky, D. J. Nat. Cell. Biol. 9, 1102-1109 (2007).

2. Gatica, D., Lahiri, V. \& Klionsky, D. J. Nat. Cell. Biol. 20, 233-242 (2018).

3. Mizumura, K., Choi, A. M. \& Ryter, S. W. Front. Pharmacol. 5, 244 (2014).

4. Geng, J. \& Klionsky, D. J. EMBO Rep. 9, 859-864 (2008).

5. Xie, Z., Nair, U. \& Klionsky, D. J. Mol. Biol. Cell. 19, 3290-3298 (2008).

6. Noda, N. N. et al. Genes Cells 13, 1211-1218 (2008).

7. Marshall, R. S., Hua, Z., Mali, S., McLoughlin, F. \& Vierstra, R. D. Cell. 177, 766-781. e24 (2019).

8. Marshall, R. S., Li, F., Gemperline, D. C., Book, A. J. \& Vierstra, R. D. Mol. Cell. 58, 1053-1066 (2015).

9. Tang, W. K. \& Xia, D. Front. Mol. Biosci. 3, 79 (2016). 